\title{
IS HIGH INITIAL AQUEOUS OUTFLOW OF BENEFIT IN TRABECULECTOMY?
}

\author{
M. BATTERBURY and P. K. WISHART \\ Liverpool
}

\begin{abstract}
SUMMARY
A modification of the trabeculectomy technique was designed in an attempt to increase the outflow of aqueous into the subconjunctival space in the early post-operative period. This 'high flow' method was compared with a control group of eyes undergoing a standard trabeculectomy. Fifty-three eyes of 44 patients were recruited to this study. Follow-up was for a minimum of 12 months (mean 20 months). The high flow method resulted in lower intraocular pressures (IOP) on days 1 and 2 and shallower anterior chambers without increased incidence of conjunctival wound leak, suggesting that high outflow was indeed achieved. However, long-term control of IOP was not better than that achieved by the conventional technique. IOP control (IOP $<21 \mathrm{mmHg}$ ) was achieved in $64.2 \%$ of eyes (34 eyes) without additional topical medication. IOP control with topical medication was satisfactory in the remaining 19 eyes, and no patient required further drainage surgery. Further analysis of the data showed that the most important factor determining success or failure of trabeculectomy in controlling IOP was the duration and number of prior topical medications. The success rate for trabeculectomy was significantly higher in those eyes with a shorter duration and quantity of prior topical therapy.
\end{abstract}

Since the introduction of the trabeculectomy by Cairns ${ }^{1}$ and Watson ${ }^{2}$ there have been several attempts to modify the technique to improve on the outcome. These have included alteration of the size of the scleral flap and of the corneal block, ${ }^{3}$ alteration of the thickness of the scleral flap, ${ }^{4}$ trabeculectomy with fistula formation, ${ }^{5}$ the omission of scleral flap sutures and posterior lip cautery. ${ }^{6}$ The original procedure made use of a limbal-based conjunctival flap, ${ }^{1}$ but others have advocated the use of a fornix-based conjunctival flap. ${ }^{4,7-10}$ The theoretical advantages of the fornix-based conjunctival flap include: improved exposure and access; reduced risk of conjunctival button-hole formation; reduced incidence of thin-walled blebs; and location of the bleb more posteriorly, away from the cornea. However, no study has shown a significantly improved long-term outcome with either flap.

Correspondence to: P. K. Wishart, FCOphth, St. Paul's Eye Unit, Royal Liverpool University Hospital, Prescot St., Liverpool L7 8XP, UK.
With a trabeculectomy with a fornix-based conjunctival flap, there is routinely escape of aqueous at the cut edge of the conjunctiva, though in most cases this resolves spontaneously. ${ }^{11}$ Because of this leak, most surgeons would be reluctant to suture loosely a scleral flap under a fornixbased conjunctival flap, in case gross leakage occurred, leading to prolonged hypotony and anterior chamber shallowing. Thus most surgeons prefer to reduce this risk by tightly suturing the scleral flap to limit egress of aqueous into the subconjunctival space. Savage et al. ${ }^{12}$ advocated this technique and manipulated post-operative aqueous outflow by selective laser lysis of scleral flap sutures.

The aim of this study was to determine whether a technique allowing high initial aqueous outflow could provide better long-term control of intraocular pressure without adverse short-term sequelae than the standard technique of a tightly sutured scleral flap under a fornix-based conjunctival flap. The high flow technique consisted of a loosely sutured triangular-shaped scleral flap under the protection of a limbal-based conjunctival flap.

\section{PATIENTS AND METHODS}

Patients requiring trabeculectomy for control of intraocular pressure (IOP) were recruited consecutively to this study. The indication for surgery in all patients was an IOP elevated above $21 \mathrm{mmHg}$ despite maximum tolerated medical therapy in the presence of glaucomatous disc damage and visual field loss. Patients who had previously undergone intraocular surgery were not recruited, so the conjunctiva in all cases was undisturbed. Also excluded were patients who had had inflammatory eye disease. The study was designed to follow patients for a minimum of 12 months.

The duration of topical therapy and number of topical antiglaucoma medications in use at the time of surgery were recorded. Eyes were randomly allocated to one of two groups.

\section{High Flow Technique (Fig. 1a)}

Following dissection of a limbal-based conjunctival flap, a triangular-shaped partial-thickness scleral flap was fashioned. The corneal block was excised and a peripheral ïri- 


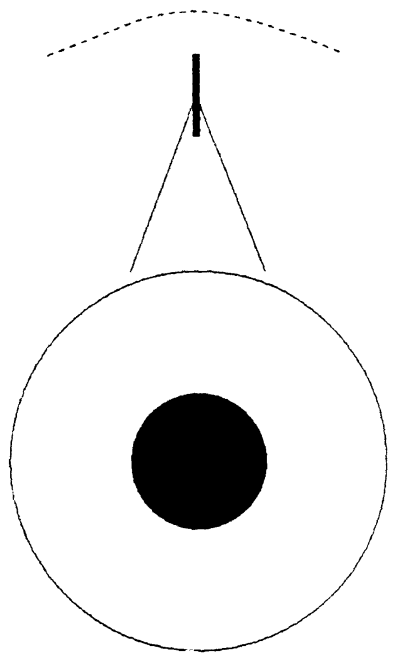

Fig. 1 (a). High flow technique.

dectomy performed. The scleral flap was replaced with a single loosely tied $8 / 0$ vicryl or virgin silk suture. Tenon's capsule was sutured with continuous $8 / 0$ vicryl, and the conjunctival wound edges apposed with interrupted 8/0 vicryl or virgin silk, to achieve a water-tight closure.

\section{Low Flow Technique (Fig. 1b)}

Conjunctiva and Tenon's capsule were divided immediately behind the limbus and reflected back, producing an incision of short arc length. A square-shaped partial-thickness scleral flap was made. The corneal block was excised, and a peripheral iridectomy performed. The scleral flap was then replaced with four tightly tied $8 / 0$ vicryl or virgin silk sutures, one at each posterior corner and one on either side close to the limbus. The conjunctival wound was repaired with several $8 / 0$ vicryl or virgin silk sutures sufficient to achieve a water-tight closure.

The triangular scleral flap was chosen in the high flow technique since we wished to use one suture only, to minimise resistance to outflow. One suture into a square flap might have resulted in curling of the corners of the flap. The corneal block was of a similar size in each technique.

Patients were observed for at least 2 days post-operatively before discharge home. Careful post-operative examination included a daily search for conjunctival wound leak (Seidel test), estimation of anterior chamber depth, presence of choroidal detachment and measurement of IOP. Anterior chamber depth was classified as: deep (normal depth), shallow (iris-corneal touch, central anterior chamber present), or flat (lens-corneal touch). Follow-up was at week 1, at 3, 6, 9 and 12 months, and thereafter at 6-month intervals. Additional examinations were conducted as clinical circumstances dictated.

\section{RESULTS}

Fifty-three eyes of 44 patients were recruited to the study. Fifty-one eyes had primary open angle glaucoma and 2 had aniridia. Twenty-seven eyes were randomised into the high flow group, and 26 eyes into the low flow group. One

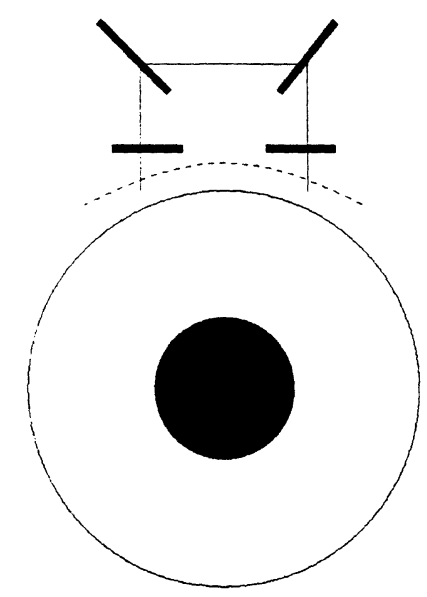

Fig. 1 (b). Low flow technique.

eye of the patient with aniridia was assigned to the low flow group, the other eye to the high flow group. Mean age and mean duration of follow-up were similar in the two groups (Table I).

\section{Early Post-operative IOP (Table II)}

High flow eyes had lower IOPs on days 1 and 2. These differences are statistically significant. However, at 1 week there was no difference.

\section{Early Post-operative Anterior Chamber Depth} (Table III)

There was a tendency for eyes in the high flow group to have shallower anterior chambers, but this difference is not statistically significant. One case of lens-corneal touch occurred, in an eye in the low flow group. This eye required anterior chamber reformation, after which it progressed satisfactorily, maintaining good central vision and adequately controlled IOP.

\section{Conjunctival Wound Edge Leak (Table IV)}

Fifteen of the low flow eyes with fornix-based conjunctival flaps showed aqueous leakage (positive Seidel test) from the conjunctival wound edge. One of these was the eye with lens-corneal touch. Only one eye in the high flow group showed aqueous leakage. This difference is statistically significant.

Table I. Patient details

\begin{tabular}{lcc}
\hline & High flow group & Low flow group \\
\hline Male/female & $12 / 15$ & $17 / 9$ \\
$\begin{array}{l}\text { Mean age (yr) } \\
\begin{array}{l}\text { Mean (range) follow-up } \\
\text { (months) }\end{array}\end{array}$ & 64.5 & 66.5 \\
\hline
\end{tabular}

Table II. Mean IOP $(\mathrm{mmHg})$ in the early post-operative period

\begin{tabular}{lccc}
\hline & Day 1 & Day 2 & Week 1 \\
\hline High flow group & 5.0 & 4.5 & 11.6 \\
Low flow group & 7.5 & 7.2 & 11.8 \\
$p$ & $<0.05$ & $<0.05$ & $>0.05$ \\
\hline
\end{tabular}




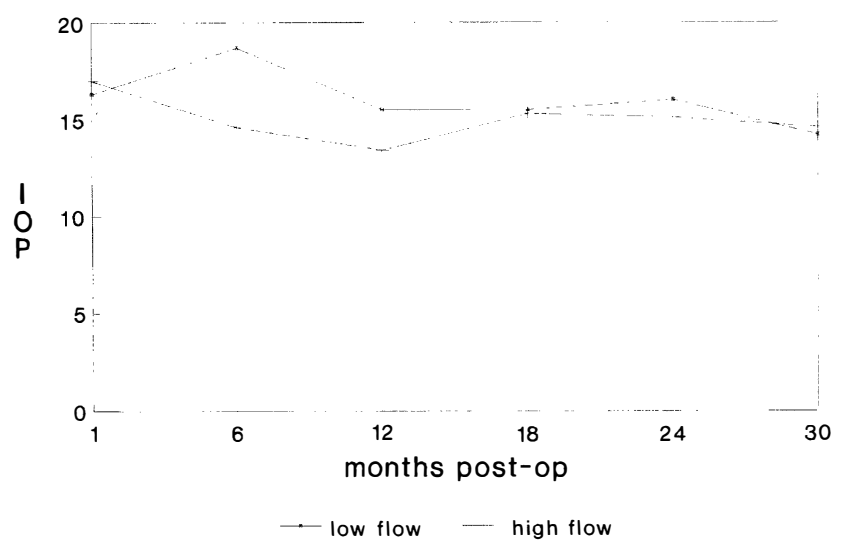

Fig. 2. Long-term IOP control.

\section{Choroidal Detachment}

Although a careful attempt was made in all subjects to examine for the presence of choroidal detachment, it was not possible to be certain of the presence or absence of this sign on clinical examination in all cases. Six eyes in the high flow group and 7 eyes in the low flow group were noted to have choroidal detachment.

\section{Bleb Morphology}

Sixteen eyes had thin-walled cystic blebs. Nine of these were in the high flow group and 7 in the low flow group.

\section{Long-Term IOP Control}

Nine eyes $(33.3 \%)$ in the high flow group required additional measures to control intraocular pressure (Table V). Ten eyes $(38.4 \%)$ in the low flow group required additional subsequent treatment. These differences are not statistically significant. Comparing the long-term IOPs of those eyes of each group which did not require additional treatment, no significant difference was detectable (Fig. 2).

\section{Influence of Prior Topical Therapy}

Trabeculectomy alone failed to control IOP in 19 of 53 eyes, and additional topical medication or repeat trabeculectomy was required (Table V). In these 19 eyes that failed, the mean duration of topical treatment and number of medications prescribed at the time of surgery were significantly greater than in those eyes in which trabeculectomy was successful (Table VI).

Table III. Anterior chamber depth

\begin{tabular}{lccc}
\hline & Deep & Shallow & Lens-corneal touch \\
\hline High flow group & 18 & 9 & 0 \\
Low flow group & 20 & 5 & 1 \\
\hline
\end{tabular}

Table IV. Conjunctival wound edge leak (Seidel test)

\begin{tabular}{|c|c|c|c|}
\hline & Absent & Present & \\
\hline High flow group & 26 & 1 & $p<0.05$ \\
\hline
\end{tabular}

\section{DISCUSSION}

Trabeculectomy has been shown to be a successful method of lowering IOP. ${ }^{13-17}$ Surgeons differ in their preference for one technique over another, particularly regarding the type of conjunctival flap. The fornix-based conjunctival flap provides better exposure, is less likely to result in formation of a conjunctival button-hole, and is said by some to produce a thicker-walled bleb located more posteriorly behind the cornea. However, with the fornix-based flap there is an increased risk of conjunctival wound edge leak which may result in hypotony and lens-corneal touch. For this reason, most surgeons suture the scleral flap tightly, thus limiting the flow of aqueous into the subconjunctival space in the early post-operative period.

This study was initiated to discover whether high initial flow into a water-tight subconjunctival space could be achieved, and whether such a technique might provide better long-term control of IOP without short-term adverse effects.

A high flow technique was designed (Fig. 1a). The high flow technique differed from the low flow technique in three respects: the type of conjunctival flap, the shape of the scleral flap and the number of scleral flap sutures. The scleral flap was secured with a single loosely tied suture to minimise resistance to outflow, but was covered by a limbal-based conjunctival flap rendered water-tight to prevent the gross leakage from the conjunctival wound edge that may occur with fornix-based flaps. ${ }^{11}$ The scleral flap was triangular so that a single suture could be applied. A single suture in a square flap would be expected to result in curling of the free corners, producing a bulky bleb. The size and shape of the scleral flap does not of itself influence the success of the surgery. ${ }^{3,9}$

This high flow technique resulted in lower IOPs on days 1 and 2 (Table II) and shallower anterior chambers (Table III), without aqueous leak (Table IV). These results suggest that a higher rate of aqueous outflow into the subconjunctival space was indeed achieved, and that this occurred without increased risk of lens-corneal touch. However, there was no improvement in long-term IOP control compared with a conventional technique (Fig. 2, Table V).

Some surgeons argue that the quality of the bleb with a fornix-based conjunctival flap is superior to that achieved with a limbal-based flap, but studies of trabeculectomy technique have not confirmed this belief. ${ }^{9,10}$ Our results similarly show no difference in bleb morphology.

Eyes in the two groups were well matched (Table I). All patients had primary open angle glaucoma, except one, who had aniridia. The two eyes of this patient were nevertheless included, since one eye was assigned to the high flow group and the other to the low flow group. The patients recruited to this study carried a good prognosis for

Table V. Success of long-term IOP control

\begin{tabular}{lcc}
\hline & $\begin{array}{c}\text { Trabeculectomy } \\
\text { alone }\end{array}$ & $\begin{array}{c}\text { Trabeculectomy + } \\
\text { topical medication }\end{array}$ \\
\hline High flow group & 18 & 9 \\
Low flow group & 16 & 10 \\
\hline
\end{tabular}


Table VI. Topical antiglaucoma medication at time of surgery

\begin{tabular}{lccrc}
\hline & $\begin{array}{c}\text { Duration of } \\
\text { therapy (months) }\end{array}$ & $\begin{array}{c}\text { No. of } \\
\text { treatments }\end{array}$ & $\begin{array}{c}\text { Beta-blocker } \\
\text { alone }\end{array}$ & $\begin{array}{c}\text { Beta-blocker }+ \\
\text { pilocarpine } \\
\text { alone }\end{array}$ \\
\hline IOP control without medication & 40 & 1.6 & 12 & 21 \\
IOP control with medication & 92 & 1.95 & 1 & 18 \\
$p$ & $<0.05$ & $<0.05$ & 0 \\
\hline
\end{tabular}

long-term success of trabeculectomy in controlling IOP: there were no blacks, no young people, no eye with previous inflammatory eye disease and no eye that had previously undergone intraocular surgery. Most studies show trabeculectomy to be an effective method of controlling IOP, and a success rate of $60-80 \%$ is usually achieved without additional topical medication. ${ }^{3,9,13-15}$ With additional medication success rates of $90-95 \%$ are achieved..$^{3,7-9,13}$ The aim of this study was to see whether a technique producing high initial aqueous outflow could improve the success rate of trabeculectomy. As a high success rate with a standard trabeculectomy was to be expected, we analysed our results with regard to long-term control of IOP without the addition of further medication, in an attempt to demonstrate a difference in success achieved by the two surgical techniques. All patients achieved satisfactory IOP control following trabeculectomy during the course of this study, although $35.8 \%$ of eyes in this study required additional topical medical therapy to achieve long-term IOP control (Table V). In no case was further surgery necessary to achieve control.

Trabeculectomy failure is most commonly due to formation of scar tissue in the subconjunctival space. ${ }^{18}$ Most failures occur in the first few months following surgery. ${ }^{19}$ High initial outflow of aqueous into the subconjunctival space might have reduced the incidence of failure, by a flow effect maintaining the potential space between conjunctiva and sclera. However, the failure rate in the low and high flow groups was similar. Analysis of our results showed that varying the trabeculectomy technique did not alter the long-term outcome (Table V), but that a prolonged duration and increased number of topical treatments had an adverse effect on outcome (Table VI). There is increasing evidence that prior treatment with topical antiglaucoma medication causes morphological changes in conjunctiva and Tenon's capsule ${ }^{20}$ and that primary trabeculectomy is more likely to lead to prolonged IOP control than trabeculectomy after many applications of topical antiglaucoma treatment. ${ }^{17}$

\section{CONCLUSION}

It would appear that the state of the tissues around the scleral flap has a greater influence on the long-term survival of the trabeculectomy than the rate of aqueous outflow from the anterior chamber into the subconjunctival space in the early post-operative period.

Analysis of the results of this study showed that modifying the trabeculectomy technique did not improve the long-term outcome, and that neither method caused more early post-operative complications. We therefore conclude that these high and low flow trabeculectomy tech- niques are equally effective, and recommend that the surgeon choose whichever method is preferred.

Key words: Glaucoma, Glaucoma surgery, Trabeculectomy.

\section{REFERENCES}

1. Cairns JE. Trabeculectomy: preliminary report of a new method. Am J Ophthalmol 1968;66:673-9.

2. Watson PG. Trabeculectomy: a modified ab externo technique. Ann Ophthalmol 1970;2:199-205.

3. Starita RJ, Fellman RL, Spaeth GL, Poryzees EM. Effect of varying size of scleral flap and corneal block on trabeculectomy. Ophthalmic Surg 1984;15:484-7.

4. Freedman J. Flap selection in glaucoma filtration surgery. Ann Ophthalmol 1987;19:449-52.

5. Welsh NH. Trabeculectomy with fistula formation in the African. Br J Ophthalmol 1972;56:32-6.

6. Thommy CP, Bhar IS. Trabeculectomy in Nigerian patients with open angle glaucoma. Br J Ophthalmol 1979;63: $636-42$.

7. Luntz MH, Freedman J. The fornix-based conjunctival flap in glaucoma filtration surgery. Ophthalmic Surg 1980;11: 516-21.

8. Luntz MH. Trabeculectomy using a fornix-based conjunctival flap and tightly sutured scleral flap. Ophthalmology 1980;87:985-9.

9. Shuster JN, Krupin T, Kolker AE, Becker B. Limbus- v. fornix-based conjunctival flap in trabeculectomy: a long-term randomised study. Arch Ophthalmol 1984;102:361-2.

10. Traverso CE, Tomey KF, Antonios S. Limbal- vs. fornixbased conjunctival trabeculectomy flaps. Am J Ophthalmol 1987; 104:28-32.

11. Spaeth GL. Glaucoma surgery. In: Duane T, editor. Clinical ophthalmology, vol 5. Philadelphia: JB Lippincott, 1989: p 20.

12. Savage JA, Condon GP, Lytle RA, Simmons RJ. Laser suture lysis after trabeculectomy. Ophthalmology 1988;95: 1631-8.

13. Watson PG, Barnett F. Effectiveness of trabeculectomy in glaucoma. Am J Ophthalmol 1975;79:831-45.

14. Jay JL, Murray SB. Characteristics of reduction of intraocular pressure after trabeculectomy. Br J Ophthalmol 1980; 64:432-5.

15. Jerndal T, Lundstrom M. 330 trabeculectomies: a long time study. Acta Ophthalmol (Copenh), 1980;58:947-56.

16. Mills KB. Trabeculectomy: a retrospective long-term follow-up of 444 cases. Br J Ophthalmol 1981;65:790-5.

17. Lavin MJ, Wormald RPL, Migdal CS, Hitchings RA. The influence of prior therapy on the success of trabeculectomy. Arch Ophthalmol 1990;108:1543-8.

18. Maumenee AE. External filtering operations for glaucoma: the mechanism of function and failure. Trans Am Ophthalmol Soc 1960;58:319-28.

19. Inaba Z. Long-term results of trabeculectomy in the Japanese: an analysis of lifetable method. Jpn J Ophthalmol 1982; 26:361-73.

20. Sherwood MB, Grierson I, Millar L, Hitchings RA. Longterm morphologic effects of antiglaucoma drugs on the conjunctiva and Tenon's capsule in glaucomatous patients. Ophthalmology 1989;96:327-35. 